QUALITY IMPROVEMENT ARTICLE

\title{
Leveraging the Massachusetts perinatal quality collaborative to address the COVID-19 pandemic among diverse populations
}

\author{
Katherine Sullivan $\mathrm{DD}^{1 凶}$, Mandy B. Belfort $\mathbb{D}^{2}$, Patrice Melvin ${ }^{3}$, Asimenia Angelidou $\mathbb{D D}^{4,5}$, Aviel Peaceman ${ }^{6}$, Jessica E. Shui ${ }^{7}$, \\ Ruben Vaidya $\mathbb{D}^{8}$, Rachana Singh ${ }^{8,9}$, Ruby Bartolome ${ }^{6}$, Silvia Patrizi ${ }^{2}$, Neha Chaudhary ${ }^{4,10}$, Ilona Telefus Goldfarb ${ }^{11}$, Ivana Culic ${ }^{4}$, \\ Diana Yanni ${ }^{4}$, Munish Gupta ${ }^{4}$, Mark Hudak (iD ${ }^{12}$ and Margaret G. Parker ${ }^{6}$
}

(c) The Author(s), under exclusive licence to Springer Nature America, Inc. 2021

\begin{abstract}
OBJECTIVE: We leveraged the Massachusetts perinatal quality collaborative (PQC) to address the COVID-19 pandemic. Our goals were to: (1) implement perinatal practices thought to reduce mother-to-infant SARS-CoV-2 transmission while limiting disruption of health-promoting practices and (2) do so without inequities attributable to race/ethnicity, language status, and social vulnerability. METHODS: Main outcomes were cesarean and preterm delivery, rooming-in, and breastfeeding. We examined changes over time overall and according to race/ethnicity, language status, and social vulnerability from 03/20-07/20 at 11 hospitals.

RESULTS: Of 255 mothers with SARS-CoV-2, 67\% were black or Hispanic and $47 \%$ were non-English speaking. Cesarean decreased ( $49 \%$ to $35 \%$ ), while rooming-in (55\% to $86 \%$ ) and breastfeeding (53\% to $72 \%$ ) increased. These changes did not differ by race/ ethnicity, language, or social vulnerability.

CONCLUSIONS: Leveraging the Massachusetts PQC led to rapid changes in perinatal care during the COVID-19 crisis in a short time, representing a novel use of statewide $P Q C$ structures.
\end{abstract}

Journal of Perinatology (2021) 41:2674-2683; https://doi.org/10.1038/s41372-021-01136-0

\section{INTRODUCTION}

The first surge of the SARS-CoV-2 pandemic in the United States (US) occurred from March to June 2020, with the highest prevalence in the Northeast. Hospitals rapidly changed medical care practices to accommodate the influx of patients infected with SARS-CoV-2. In the perinatal setting, hospital teams focused on implementing practices to reduce transmission of SARS-CoV-2 from mother to infant, but faced major challenges in minimizing disruption to existing health-promoting practices. During the early stages of the pandemic, population-level data that may inform optimal perinatal care practices were lacking. For example, in March 2020, the only available data on newborn transmission came from case reports from China where women with SARS-CoV2 were delivered by scheduled cesarean and separated from their infants after delivery with no direct breastfeeding. While this approach was associated with minimal to no transmission of SARS-CoV-2 to newborn infants [1], it was unclear whether these practices were essential to prevent transmission, or if a similar low risk of transmission could be achieved with perinatal care practices with established health benefits to both mothers and infants [2], such as rooming-in and direct breastfeeding.
Statewide perinatal quality collaboratives $(\mathrm{PQC})$ are networks of perinatal care providers and public health agencies that collaborate to improve outcomes of mother-infant dyads through sharing of ideas, data, and quality improvement $[3,4]$. When the SARS-CoV-2 pandemic hit Massachusetts, we leveraged our existing PQC structure to optimize perinatal care for women who tested positive for SARS-CoV-2 and their infants for three main reasons. First, this forum enabled sharing and collaboration among centers to facilitate rapid perinatal practice improvement. Second, $\mathrm{PQCs}$ can be most effective when addressing topics with considerable variability in practices [4], which we confirmed after conducting a short survey among clinical leaders of maternity units in Massachusetts from $3 /$ $31 / 20$ to $4 / 5 / 20$. For example, we found large variability in reported approaches to direct breastfeeding (60\% of hospitals encouraged breastfeeding with precautions and $28 \%$ prohibited or discouraged this practice) [5]. Lastly, we recognized that we could quickly leverage the existing data infrastructure of our PQC, which has been used to evaluate perinatal care practices across sites over time for several years [6-8].

Evidence of disparities in quality of perinatal care according to race/ethnicity $[9,10]$ and language status $[11]$ is well-documented and is partly responsible for disparities in perinatal outcomes.

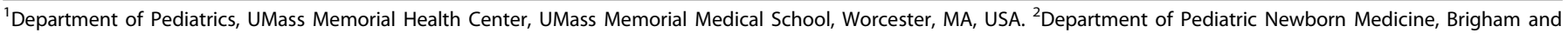

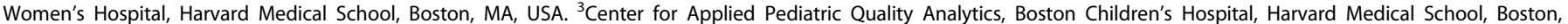

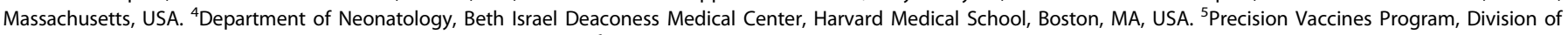

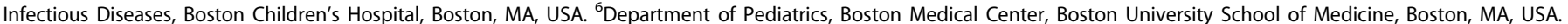





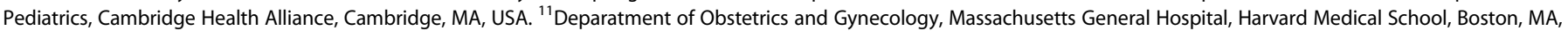
USA. ${ }^{12}$ Department of Pediatrics, University of Florida Health, University of Florida College of Medicine, Jacksonville, FL, USA. ${ }^{\infty}$ email: Katherine.Sullivan@umassmemorial.org
} 
Table 1. Perinatal COVID-19 Hospital Care Practices in Massachusetts.

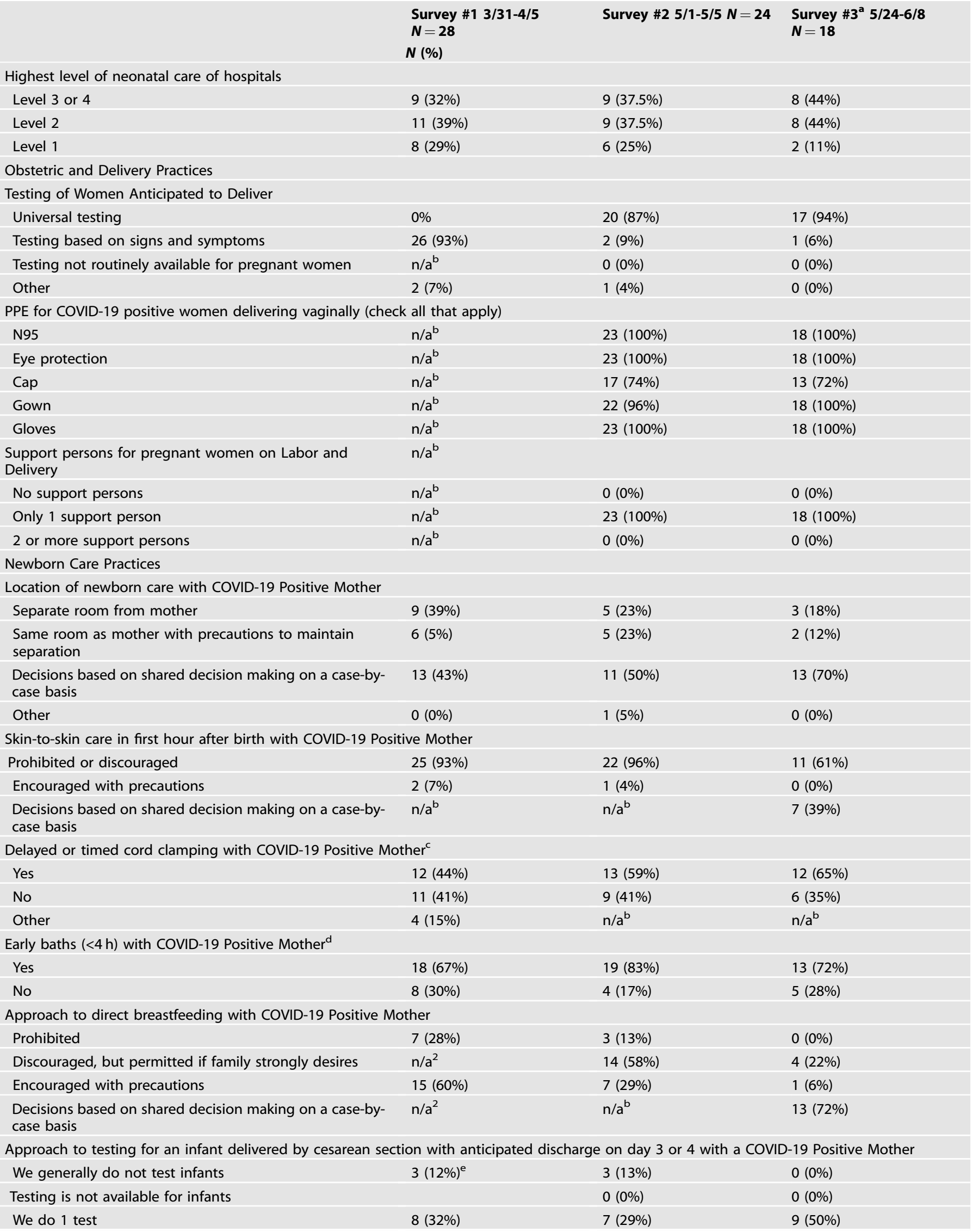


Table 1 continued

\begin{tabular}{|c|c|c|c|}
\hline & $\begin{array}{l}\text { Survey \#1 3/31-4/5 } \\
N=28 \\
N(\%)\end{array}$ & Survey \#2 5/1-5/5 $N=24$ & $\begin{array}{l}\text { Survey \#3 } 3^{a} 5 / 24-6 / 8 \\
N=18\end{array}$ \\
\hline We do 2 or more tests & $12(48 \%)$ & $8(33 \%)$ & $9(50 \%)$ \\
\hline Other & $2(8 \%)$ & $6(25 \%)$ & $0(0 \%)$ \\
\hline \multicolumn{4}{|c|}{ Discharge Processes for non-COVID-19 Positive Mother-Infant Dyads } \\
\hline \multicolumn{4}{|l|}{ Timing of Discharge } \\
\hline Timing has not really changed & $\mathrm{n} / \mathrm{a}^{\mathrm{b}}$ & $3(13 \%)$ & $2(11 \%)$ \\
\hline Some dyads are discharged early & $\mathrm{n} / \mathrm{a}^{\mathrm{b}}$ & $7(30 \%)$ & $7(39 \%)$ \\
\hline Many dyads are discharged early & $\mathrm{n} / \mathrm{a}^{\mathrm{b}}$ & $13(57 \%)$ & $9(50 \%)$ \\
\hline $\begin{array}{l}\text { All dyads discharge early unless a medical } \\
\text { contraindication }\end{array}$ & $\mathrm{n} / \mathrm{a}^{\mathrm{b}}$ & $0(0 \%)$ & $0(0 \%)$ \\
\hline
\end{tabular}

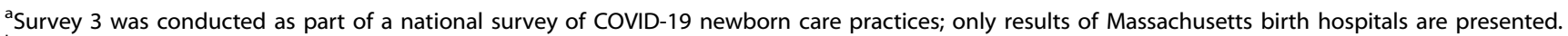

${ }^{\mathrm{b}}$ This answer choice was not available for this survey.

In survey \#2 we ascertained that 92\% of hospitals perform delayed or timed cord clamping among non-COVID-19 dyads.

dIn survey \#3 we ascertained that $0 \%$ of hospitals perform early baths among non-COVID-19 dyads.

e In survey \#1 we did not differentiate these responses.

Perinatal quality improvement may lead to inadvertent widening or persistence of social disparities [12, 13]. For these reasons, tracking quality measures according to key disparity metrics, such as race/ethnicity, language status, and other social factors is recommended in order for local teams and statewide $P Q C$ leadership to adjust their current improvement strategies and/or trial new strategies, if needed, to ensure equitable improvement across all groups $[12,13]$. Considering the early evidence in March 2020 that the SARS-CoV-2 pandemic was disproportionately impacting low-income non-Hispanic Black and Hispanic populations, we also aimed to ensure that social factors did not influence implementation of best practices.

In March 2020, the beginning of the SARS-CoV-2 pandemic surge in Massachusetts, we leveraged our PQC to achieve the following project goals: (1) implement perinatal hospital practices thought to reduce mother-to-infant SARS-CoV-2 transmission while limiting disruption of existing health-promoting practices and (2) do so without inequities attributable to race/ethnicity, language status, and social factors. We assessed our outcomes by two complementary data collection strategies. First, through monthly hospital-level surveys of perinatal care practices and second, through individual-level data collection among 11 hospitals that cared for patients living in geographic areas most impacted by SARS-CoV-2 infection [14].

\section{METHODS \\ Population and setting}

The Massachusetts Perinatal Neonatal Quality Improvement Network (PNQIN) has conducted several initiatives in the last decade with participation in one or more projects among $>80 \%$ of its 43 birth hospitals. In this project, hospitals created multi-disciplinary teams and participation was voluntary. State stakeholders included the Department of Public Health, Bureau of Family Health and Nutrition, Betsy Lehman Center for Patient Safety [15], and the Institute for Health and Recovery [16].

We collected data in two ways to understand changes in perinatal practices over time. First, we collected data on hospital-level perinatal care practices by administering three brief $(\sim 5-7 \mathrm{~min})$ electronic surveys to clinical leaders previously involved in active PNQIN projects. These surveys were conducted approximately monthly, from March 31-April 5 (survey \#1), May 1-5 (survey \#2), and May 26-June 8 (survey \#3). The surveys were sent to a single clinical leader at each hospital and ascertained current approaches to perinatal care.

To complement the brief hospital practice surveys, a subset of birthing hospitals ( 7 level 3 and 4 level 2 neonatal care facilities) collected data at the individual mother-infant dyad level by abstracting demographic and clinical data from electronic medical records. These hospitals served patients that lived in cities and towns with high COVID-19 transmission rates. We collected data among women who delivered between March 1 and July 31, 2020 using the American Academy of Pediatrics (AAP) National Perinatal COVID-19 Registry (NPC-19) complemented by a limited Massachusetts-specific perinatal COVID-19 data set. Similar to NPC-19, we included any infant born to a mother with positive SARS-CoV-2 testing 14 days before until 3 days after birth and collected data on maternal severity of illness, birth characteristics, infant SARS-CoV-2 testing during the birth hospitalization, and hospital practices. The Massachusetts data set additionally captured maternal zip code and language status, week of birth, and 30-day postdischarge infant SARS-CoV-2 testing. Both datasets were approved by local institutional review boards of the 11 participating hospitals that contributed dyad-level data and were deemed minimal risk and exempt.

\section{Measures}

Hospital-level data captured snapshots of guidelines for maternal and infant SARS-CoV-2 testing procedures, types of personal protective equipment used by neonatal providers at delivery, and approaches to location of care, breastfeeding, skin-to-skin care in the first hour of life, delayed or timed cord clamping, early baths and newborn discharge.

Individual dyad-level data included socioeconomic variables, including maternal race/ethnicity, which we categorized as non-Hispanic white, nonHispanic black, non-Hispanic Asian, Hispanic of any race, and all others; and maternal language status, which we categorized as English, Spanish, Portuguese or other non-English speaking. We converted zip codes to census tracts using the U.S. Housing and Urban Development's "Crosswalk Files," which is endorsed by the Office of Policy Development and Research [17]. In this approach, when a zip code represents more than one census tract, the census tract with the highest residential population is chosen. Census tract was then used to determine degree of social vulnerability according to the Centers for Disease Control (CDC) Social Vulnerability Index (SVI) [18]. In this index, 15 census variables are used to characterize overall social vulnerability and subscales, which include socioeconomic status, household composition, minority status/language, and housing type. We defined "social vulnerability" as >90th percentile on the SVI, according to CDC designated definitions.

Severity of maternal illness with SARS-CoV-2 was categorized as symptomatic vs asymptomatic. We examined plurality, mode of delivery, preterm delivery ( $<37$ weeks' gestation), and infant weight, sex, and any positive SARS-CoV-2 testing during the birth hospitalization or 30-day postdischarge period. Regarding hospital practices, location of care was categorized as rooming-in at any time vs. complete separation. We categorized infant feeding as any direct breastfeeding vs. none (formula, donor milk, or expressed milk). In this analysis, we targeted direct breastfeeding only, rather than direct breastfeeding or expressed milk given by another way (e.g., bottle, syringe) because of concerns of motherto-infant SARS-CoV-2 transmission via contact through the process of direct breastfeeding, rather than consumption of expressed milk, where contact was substantially reduced [19]. 
Table 2. Characteristics of 250 mothers with peripartum positive SARS-CoV-2 testing and 255 infants born $3 / 1 / 20$ to $7 / 31 / 20$ in Massachusetts.

\section{Participating Hospitals, $\boldsymbol{n}$ (\%)}

Baystate Medical Center

Beth Israel Deaconess Medical Center 20

31

7.8

Beverley Hospital

Boston Medical Center

Brigham and Women's Hospital

Cambridge Health Alliance

Mass General Hospital

Newton Wellesley Hospital

Tufts Medical Center

UMass Memorial Medical Center

Winchester Hospital

Total

Month of birth, $n(\%)$

$\begin{array}{lll}\text { March } & 17 & 6.7 \\ \text { April } & 79 & 31.0 \\ \text { May } & 88 & 34.5 \\ \text { June } & 55 & 21.6 \\ \text { July } & 16 & 6.3\end{array}$

Maternal demographics

Age, mean (SD)

Race/ethnicity, $n$ (\%)

Hispanic (any race)

Non-Hispanic White

Non-Hispanic Black

Non-Hispanic Asian

Non-Hispanic Other

Maternal language, $n$ (\%)

English

Spanish

Portuguese

Other

Social vulnerability index ${ }^{a}$ themes; percentile ranking, mean (SD)

\begin{tabular}{|c|c|c|}
\hline Socioeconomic status & 65.8 & 23.3 \\
\hline Household composition & 53.3 & 29.4 \\
\hline Minority and language status & 71.6 & 25.6 \\
\hline Housing and transportation & 71.5 & 22.0 \\
\hline Overall & 70.2 & 23.8 \\
\hline $\begin{array}{l}\text { Social vulnerability index overall }>90 \text { th percentile, } \\
n(\%)\end{array}$ & 68 & 26.7 \\
\hline \multicolumn{3}{|l|}{ Severity of maternal illness, $n$ (\%) } \\
\hline Asymptomatic & 174 & 68.2 \\
\hline Symptomatic & 81 & 31.8 \\
\hline Sick at home before admission & 57 & 70.4 \\
\hline $\begin{array}{l}\text { Required hospitalization for treatment of } \\
\text { COVID-19 }\end{array}$ & 24 & 29.6 \\
\hline Multiple gestation, $n$ (\%) & 10 & 3.9 \\
\hline \multicolumn{3}{|l|}{ Disposition, $n$ (\%) } \\
\hline Home & 253 & 99.2 \\
\hline Transfer & 2 & 0.01 \\
\hline
\end{tabular}

\begin{tabular}{|c|c|c|}
\hline \multicolumn{3}{|l|}{ Participating Hospitals, $\boldsymbol{n}(\%)$} \\
\hline Deaths & 0 & 0 \\
\hline \multicolumn{3}{|l|}{ Infant characteristics } \\
\hline Liveborn, $n$ (\%) & 255 & 100.0 \\
\hline Gestational age, weeks, mean (SD) & 37.9 & 2.6 \\
\hline Birthweight, grams, mean (SD) & 3116.3 & 655.6 \\
\hline Female sex, $n(\%)$ & 131 & 51.4 \\
\hline \multicolumn{3}{|l|}{ Disposition } \\
\hline Home & 247 & 96.9 \\
\hline Transfer & 7 & 2.8 \\
\hline Died & 1 & 0.4 \\
\hline $\begin{array}{l}\text { Any SARS-CoV- } 2 \text { test during the newborn } \\
\text { hospitalization, } n(\%)\end{array}$ & 225 & 88.2 \\
\hline Any positive newborn test (among tested) & 5 & 2.2 \\
\hline $\begin{array}{l}\text { Any SARS-CoV- } 2 \text { test during the } 30 \text { days post- } \\
\text { discharge }{ }^{b}, n(\%)\end{array}$ & 7 & 4.6 \\
\hline Any positive newborn test (among tested) & 1 & 14.3 \\
\hline \multicolumn{3}{|l|}{$\begin{array}{l}\text { Positive SARS-CoV- } 2 \text { test during the hospitalization } \\
\text { or } 30 \text { days post-discharge }\end{array}$} \\
\hline Any positive newborn test (overall) & 6 & 2.4 \\
\hline Any positive newborn test (among tested) & 6 & 2.7 \\
\hline \multicolumn{3}{|l|}{ Main Outcomes } \\
\hline \multicolumn{3}{|l|}{ Mode of delivery, $n$ (\%) } \\
\hline Vaginal & 142 & 55.7 \\
\hline Cesarean birth & 113 & 44.3 \\
\hline Preterm (<37 weeks), $n(\%)$ & 53 & 20.8 \\
\hline $\begin{array}{l}\text { Spontaneous or augmented following initiation } \\
\text { of labor }\end{array}$ & 15 & 28.3 \\
\hline Medically-indicated & 38 & 71.7 \\
\hline \multicolumn{3}{|l|}{ Location of care, $n(\%)$} \\
\hline Any rooming-in & 167 & 65.5 \\
\hline $\begin{array}{l}\text { Complete separation during entire } \\
\text { hospital course }\end{array}$ & 88 & 34.5 \\
\hline $\begin{array}{l}\text { Any direct breastfeeding during the } \\
\text { hospitalization }\end{array}$ & 152 & 59.6 \\
\hline
\end{tabular}

${ }^{a}$ Social vulnerability index uses 15 US census variables derived from zip codes and was derived by the Centers of Disease Control.

${ }^{\mathrm{b}}$ Among 151 infants with 1 or more encounter.

We derived our main outcomes from individual dyad-level data and sought to capture perinatal hospital practices suspected of increasing mother-to-infant SARS-CoV-2 transmission at the start of our quality initiative. In March 2020, there were concerns that vaginal delivery, rooming-in and direct breastfeeding may increase the likelihood of mother-to-infant SARS-CoV-2 transmission due to close physical con tact [19]. We also hypothesized that preterm delivery may lead to increased transmission in the context of immature immune systems. We additionally examined outcomes according to severity of maternal illness (symptomatic vs. asymptomatic) and important social factors, including maternal underrepresented minority (URM) status (non-Hispanic black or Hispanic vs. all others), maternal language status (English vs. non-English) and significant social vulnerability ( $\mathrm{SVI}>90$ th percentile vs. <90th percentile), as we hypothesized that these factors may impact perinatal practices over time.

\section{QI approach}

Local multi-disciplinary teams met frequently, developed and updated guidelines on perinatal care, and trialed interventions within their home institutions. We hosted 14 obstetrical and newborn-oriented webinars every 1-3 weeks for all state participants from April to June 2020 [20, 21]. 


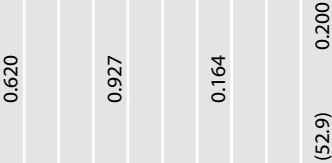

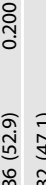

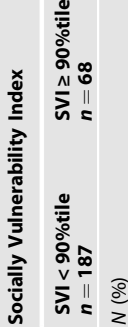

ฟิ

in

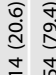

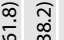

₹

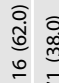

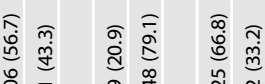

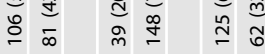

$\stackrel{\infty}{\circ} \stackrel{0}{\circ}$

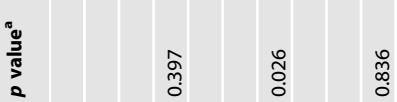

2)

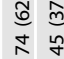

赵

₹ वे

党

ڤึ

$\bar{a}=$

ถู่

영

åd

$\widehat{\kappa}$

के

สู ఫ

ஸิ

$\stackrel{\infty}{\wedge}$

ำ

喜

$\frac{\mathrm{m}}{2}$


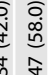

\section{กุ้ กิ่}

m

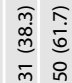

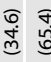

We provided updates on recent publications, emerging data from our own state, existing professional guidance and education on social disparities regarding COVID-19. Several teams shared their experience and/or posted local clinical guidelines on our website which fostered collaboration. We presented findings of our monthly practice surveys and summarized demographic and clinical characteristics of mothers and infants with positive SARS-CoV-2 tests.

\section{Statistical approach}

We examined hospital-level practices and described demographic, socioeconomic, and clinical characteristics of individual mother-infant dyads using frequencies (percent) for categorical variables and mean (SD) for continuous variables. We examined differences in our main outcome measures (cesarean and preterm delivery, location of care and direct breastfeeding) according to maternal illness severity, URM and language status, and SVI using Cochran-Mantel-Haenszel $X 2$ tests to account for clustering by hospital. Statistical process control charts ( $p$-charts) were used to analyze changes over time for our main outcomes. Standard rules were used to identify special cause variation, including eight points above or below the center line, one point beyond outer control limits, and six consecutive points increasing or decreasing [22]. When special cause variation was seen that corresponded to practice changes and was expected to persist, the center lines were shifted. We further examined our main outcomes over time according to severity of maternal illness, maternal URM and language status and SVI using run charts. Due to smaller sample sizes, we collapsed our data to 8 points. A sensitivity analysis of the hospital-level practices among the 11 centers that provided individual-level data showed findings similar to the larger group of hospitals and we therefore present the data from the larger group. All descriptive and statistical analyses were performed using SAS version 9.4 (SAS Institute, Inc, Cary, NC). QI Macros 230 for Excel (KnowWare International, Inc.) was used to create the p-charts.

\section{RESULTS}

The median number of individual providers and hospitals that participated in our webinars was 106 (range 43-177) and 26 (range 10-32), respectively. Results of our hospital-level practice surveys administered to clinical leaders at 28 hospitals that were actively involved in a PNQIN project at the time are shown in Table 1. Twenty-eight (100\%) responded to survey \#1 (early April), $24(86 \%)$ responded to survey \#2 (early May), and 18 (64\%) responded to survey \#3 (early June). Some practices remained consistent over time, such as the use of N95 masks for neonatal staff and restriction to a single support person for pregnant women at delivery (100\% throughout the survey periods). Other practices changed markedly. For example, universal testing for asymptomatic women anticipated to deliver increased from $0 \%$ in April to $87 \%$ in May and $95 \%$ in June. Mother-infant separation was reported as the preferred approach for $39 \%$ of hospitals in April but decreased to $23 \%$ in May and $18 \%$ in June. The preferred approach to skin-to-skin care after delivery was reported as prohibited or discouraged in $>90 \%$ of hospitals in April and May but this decreased to $61 \%$ by June. We found that shared decision making was increasingly reported across survey time points as the preferred approach for location of care and direct breastfeeding.

Characteristics of 255 dyads with hospital data and 151 dyads with any post-discharge infant data are shown in Table 2 . Among mothers, the majority were URMs (48.2\% were Hispanic and $18.8 \%$ were non-Hispanic Black), $46 \%$ did not speak English and $27 \%$ had an overall SVI of $>90$ th percentile, indicating significant social vulnerability. Thirty-one percent had symptomatic infection. Five infants had one or more positive SARS-CoV-2 tests among the 225 that were tested during the hospital period and 1 had a positive test in the 30-day post-discharge period, such that the overall rate of infant positive testing was $2.7 \%$ among those tested. Among the 6 infants with positive tests, 1 occurred in March and 5 in April. With respect to our main outcomes, 113/255 (44\%) of infants were born by cesarean delivery, 53/255 (21\%) were born preterm (of which $15 / 53$ [28\%] were spontaneous and $38 / 53$ [72\%] were 

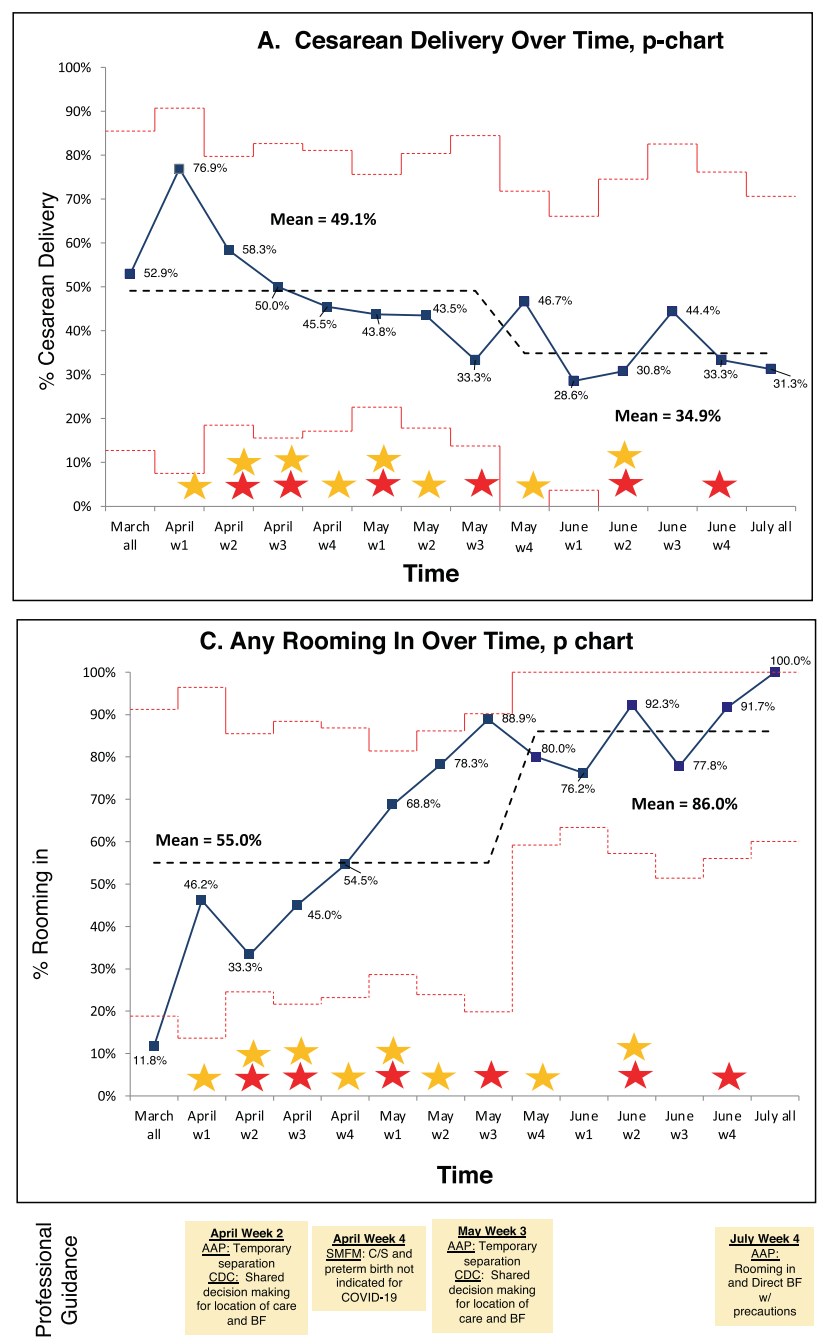

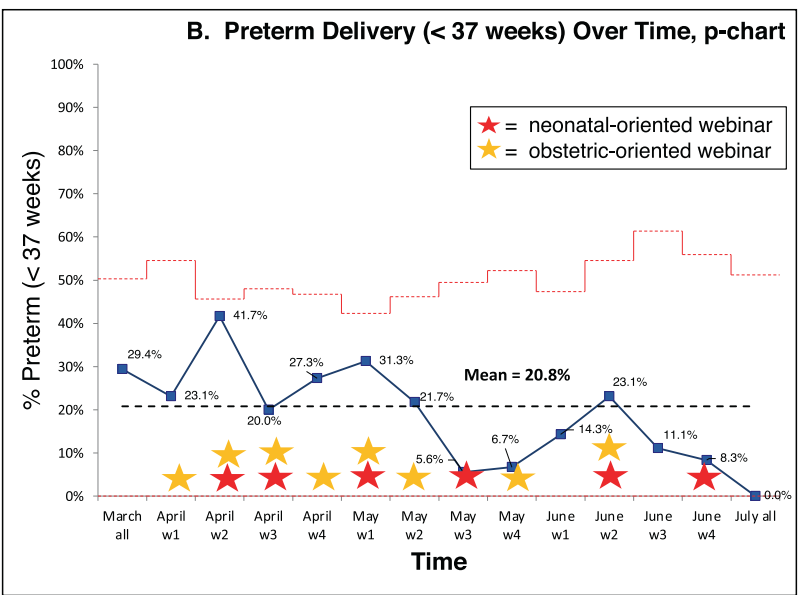

D. Any Direct Breastfeeding Over Time, $p$ chart
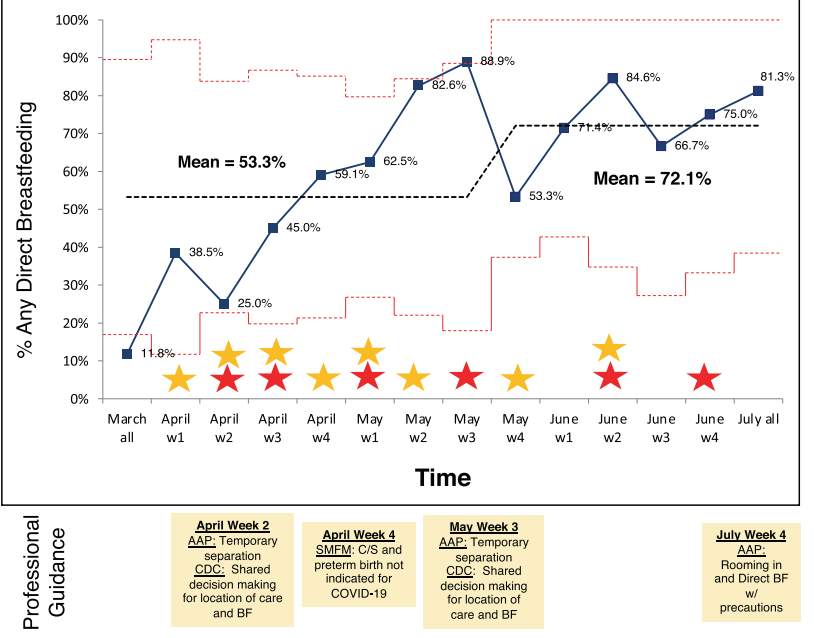

Fig. 1 AAP = American Academy of Pediatrics; CDC $=$ Centers for Disease Control; SMFM $=$ Society for Maternal Fetal Medicine; BF $=$ breastfeeding; Results of 255 mothers with positive SARS-CoV-2 testing between 14 days before until 3 days after delivery at 11 Massachusetts birthing hospitals that gave birth from March 1 to July 31, 2020. Average percent of A cesarean delivery, B preterm delivery ( $<37$ weeks), $\mathbf{C}$ any rooming-in, and $\mathbf{D}$ any direct breastfeeding are shown over time with 2 standard deviations. Red stars represent dates of newborn-oriented webinars and orange stars represent dates of obstetric-oriented webinars.

medically-indicated), 167/255 (66\%) experienced any rooming-in and 152/255 (60\%) were directly breastfed.

The relationships between our main outcomes and maternal illness severity and social factors are shown in Table 3. Symptomatic SARS-CoV-2 infection was associated with increased cesarean and preterm delivery and decreased rooming-in and direct breastfeeding, compared to asymptomatic infection $(p<$ 0.05 ). There were no significant relationships between social factors and our main outcomes, apart from language status, where we found that non-English speaking mothers had higher rates of preterm delivery (25\%), compared to English speaking mothers (17\%) $(p=0.03)$.

Control chart analysis of our main outcomes from March until July (Fig. 1) showed that cesarean delivery decreased from 49 to $35 \%$, rooming-in increased from 55 to $86 \%$, any direct breastfeeding increased from 53 to $72 \%$, and preterm delivery did not change. We did not see differences in time trends according to maternal race/ethnicity, language status or SVI (Figs. 2-5).

\section{DISCUSSION}

The majority of birthing hospitals in Massachusetts participated in a perinatal quality initiative focused on care of mothers with SARS-
CoV-2 infection and their infants during the initial surge of the pandemic. Data from 255 mother-infant dyads collected at 11 centers that cared for patients living in cities with the highest proportion of COVID-19 in Massachusetts demonstrated that rates of cesarean delivery significantly decreased and rates of roomingin and direct breastfeeding significantly increased from March to July 2020. While SARS-CoV-2 infection disproportionately affected URM, non-English, and socially vulnerable mothers, we did not find that these factors influenced how perinatal care practices changed over time during the health crisis. Hospital-level survey data showed that hospitals increasingly adopted a shareddecision making model for rooming-in and direct breastfeeding over time.

Our project highlights the strengths of leveraging statewide PQC infrastructure to support local hospitals during a public health crisis. PQCs are strongly endorsed by the Centers for Disease control and have rapidly grown in the past decade [23]. PQCs have been used for a wide array of clinical improvements and have been shown to be more effective than local site QI at facilitating sustained improvement [24, 25]. This likely occurs because PQCs provide an infrastructure for well-defined data collection and reporting, team sharing, and resources such as training in basic QI methods. In our case, Massachusetts perinatal 

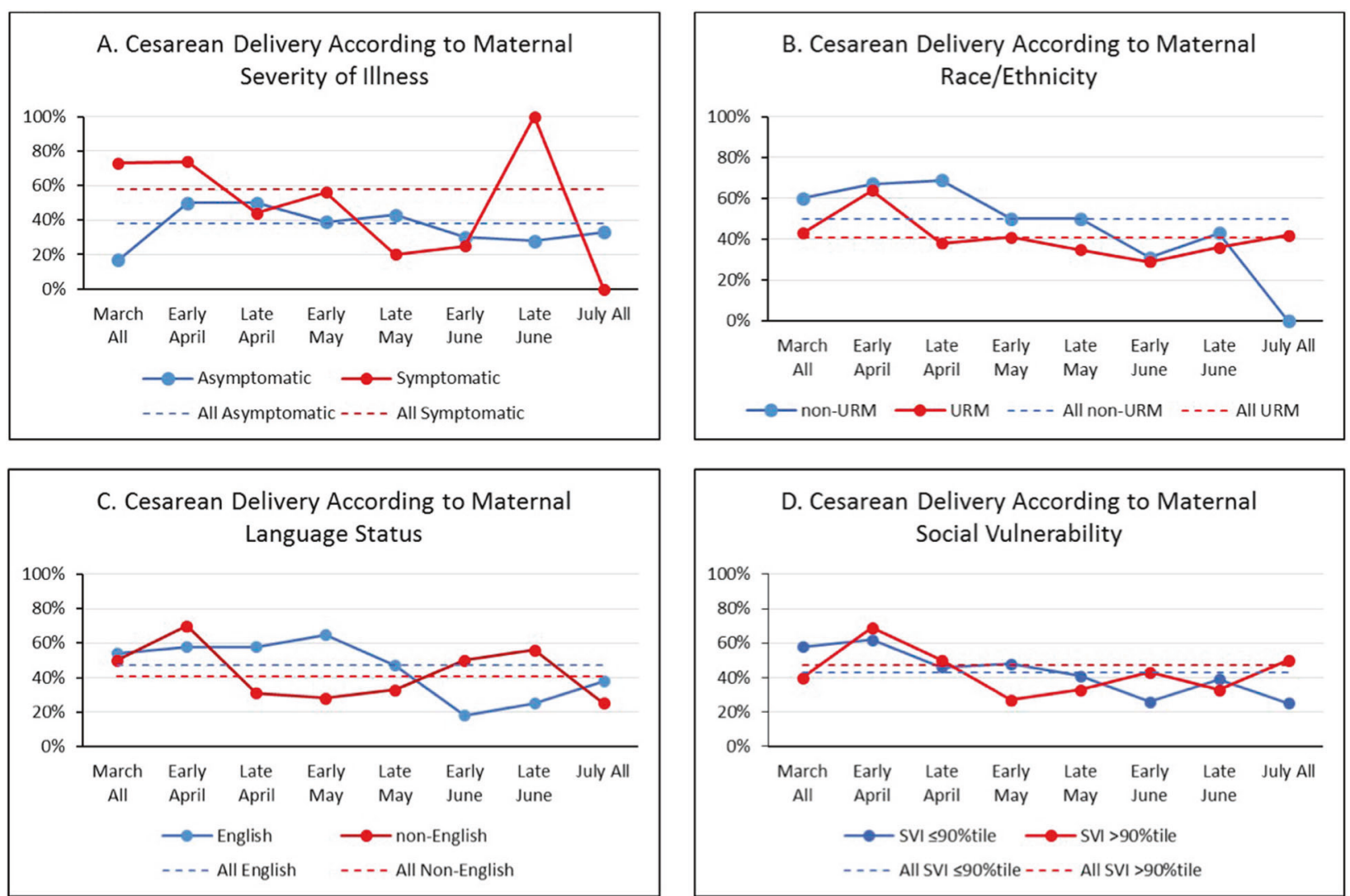

Fig. 2 URM = underrepresented minority (non-Hispanic black or Hispanic of any race); average percent of cesarean delivery over time according to maternal. A severity of illness, B race/ethnicity, C language status, and D social vulnerability index are shown.

hospital teams were accustomed to voluntary sharing of local improvement approaches, completing practice surveys, and contributing data to a central database, which existed as part of our PQC infrastructure. These resources allowed for rapid initiation and conduct of a quality initiative focused on perinatal SARS-CoV2 hospital practices. A notable difference between this initiative and previous ones was the lack of knowledge regarding evidencebased perinatal practices associated with optimal health and wellbeing of mother-infant dyads with SARS-CoV-2 at the start of our initiative, which evolved over time. In contrast, previous initiatives have targeted adoption of hospital practices with a more robust evidence base. Instead, best practice was constantly being sought and shared collaboratively by the group. Overall, we believe that our project demonstrated the successful use of the PQC structure during the time of an uncertain health crisis, which expands upon the previously reported uses of $\mathrm{PQCs}$, and further strengthens the rationale for policy support as well as funding to develop and maintain such organizations by diverse stakeholders vested in perinatal care.

We speculate that the rapid changes in perinatal care practices occurred for several reasons. First, we openly discussed emerging evidence and its implications on perinatal care at our webinars. For example, small case reports from China in March reported low rates of positive SARS-CoV-2 infant testing following cesarean delivery and immediate mother-infant separation [26, 27], however, by April, case series began to be published in Europe in which rates of positive SARS-CoV-2 infant testing was also low following vaginal delivery and rooming-in [28, 29]. By May and June, larger studies and reviews of multiple case series further affirmed that positive SARS-CoV-2 infant testing appeared to be low irrespective of delivery mode, location of care and/or direct breastfeeding [30, 31]. Second, at a webinar in May 2020, we reviewed cases of positive infant SARS-CoV-2 testing in
Massachusetts. Similar to published literature [30, 31], infants with positive tests in our state appeared to be rare [20]. Third, we discussed professional guidelines, including discrepancies (e.g., AAP $[32,33]$, CDC $[34,35]$, and Society for Maternal Fetal Medicine [36]) extensively. The first official guidance by the SMFM was published on April 30 [36], which stated that COVID-19 was not an indication for delivery among asymptomatic and mildly symptomatic women and that mode of delivery should be based on routine indications. While decision making about mode of delivery is driven by many factors, we speculate that this SMFM guidance additionally contributed to the decrease in rates of cesarean deliveries we observed. The AAP and CDC guidance, while slightly different in emphasis, did not change during the project period. In April and May 2020, the AAP guidance recommended temporary separation of mothers and infants as the safest course of action if possible and the CDC recommended use of a shared-decision making approach for location of care and direct breastfeeding. Our monthly hospital practice surveys showed increased adoption of the shared-decision making approach between April and June 2020; we hypothesize that this additionally contributed to increases in rooming-in and direct breastfeeding. Overall, we speculate that review of emerging literature, Massachusetts cases and evolving professional organization guidance helped build consensus regarding best practices and led to the rapid implementation of such practices.

We found alarming social disparities among mothers with SARSCoV-2 infection, similar to previous US studies [37]. As we have done in our previous work [6], we assessed our outcomes as a function of several social factors to determine if these factors influenced perinatal care practices. Although social factor subgroups were small, we were reassured to see that changes in perinatal practices did not vary by social factors. Nonetheless, we recognize that unconscious racial/ethnic and other social biases 

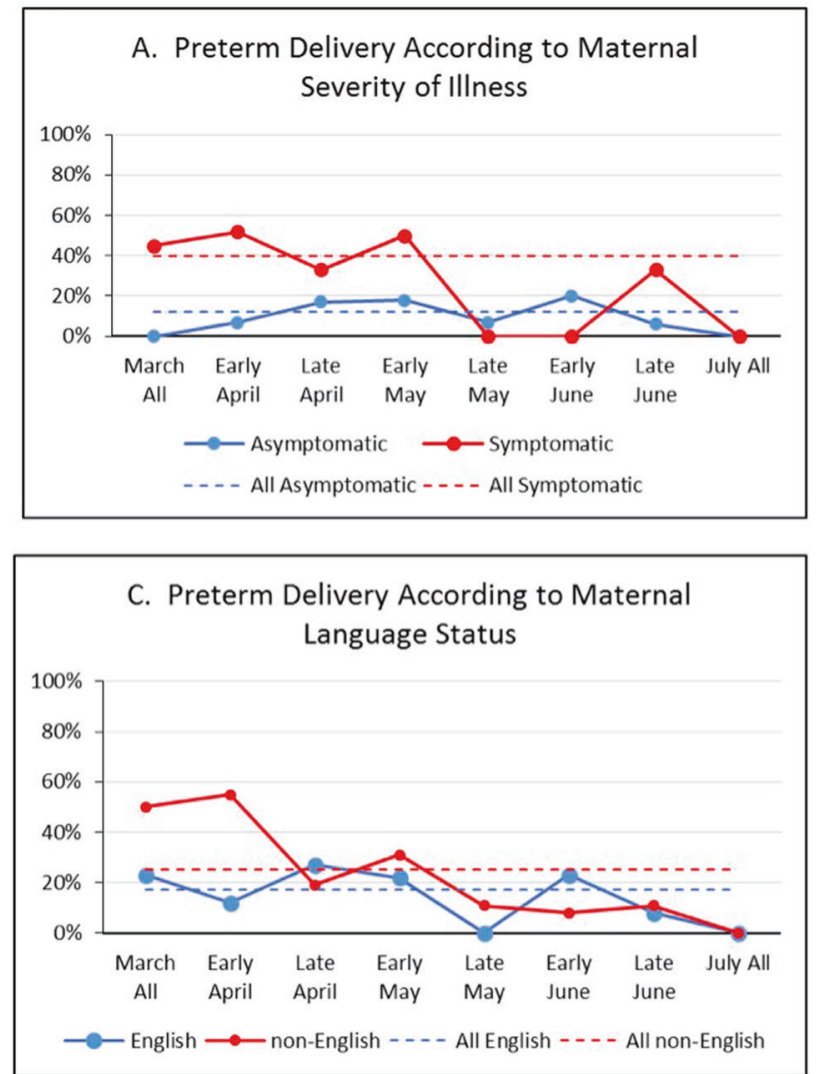

B. Preterm Delivery According to Maternal Race/Ethnicity

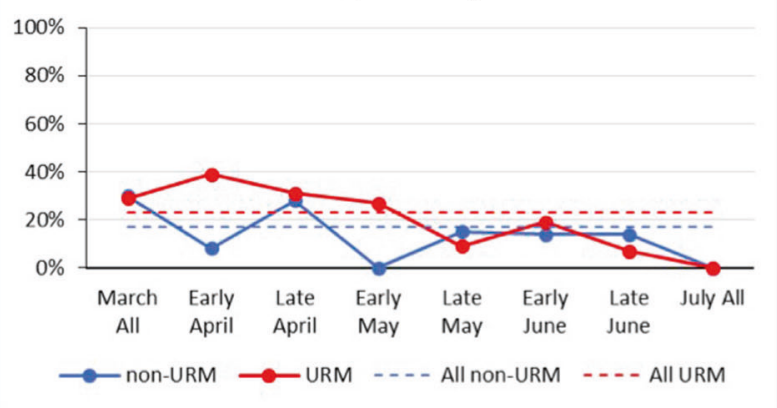

D. Preterm Delivery According to Maternal Social Vulnerability



Fig. 3 URM = underrepresented minority (non-Hispanic black or Hispanic of any race); average percent of preterm delivery (<37 weeks) over time according to maternal. A severity of illness, B race/ethnicity, C language status, and D social vulnerability index are shown.
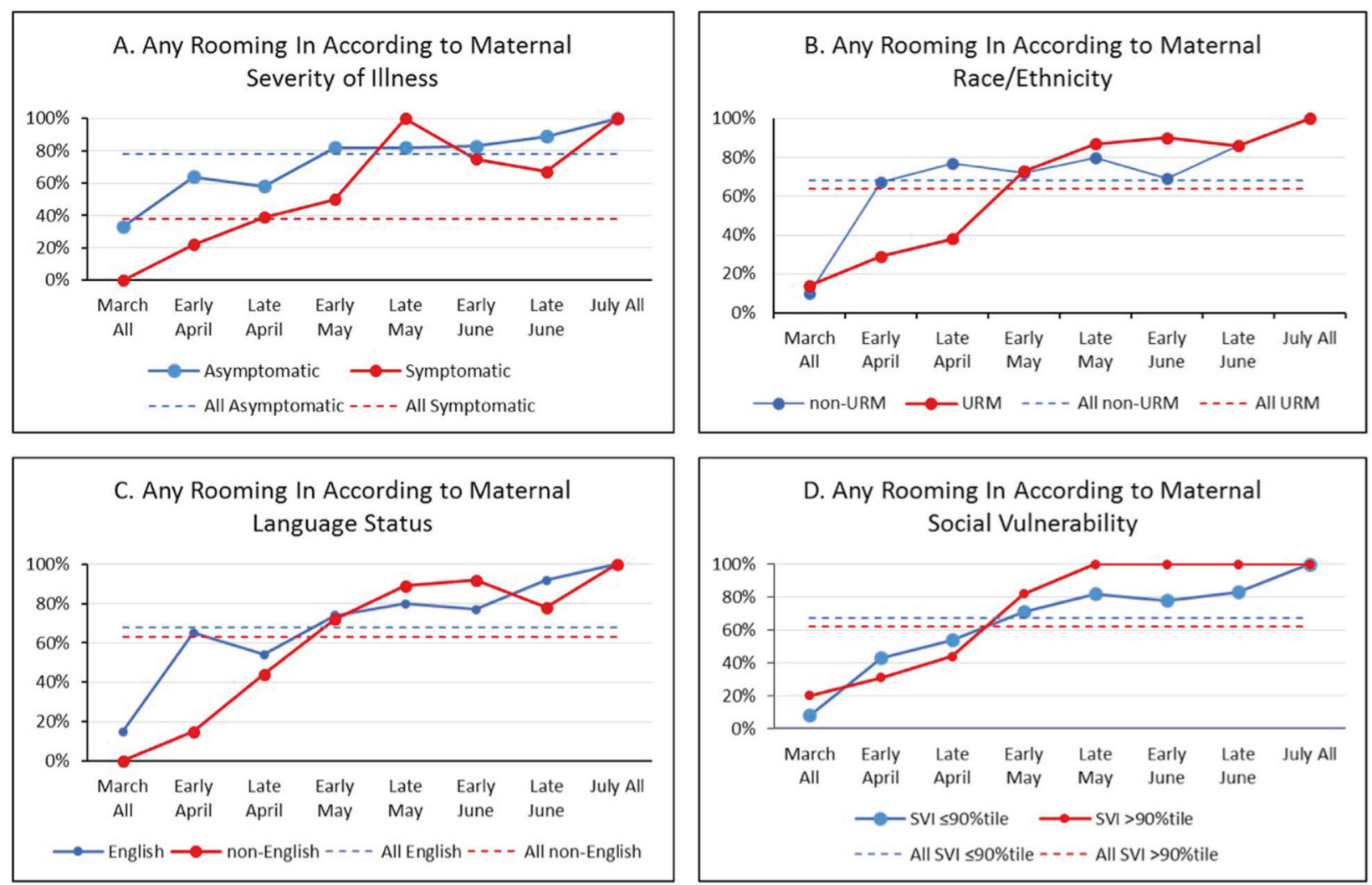

Fig. 4 URM = underrepresented minority (non-Hispanic black or Hispanic of any race); average percent of any rooming in during the newborn hospitalization over time according to maternal. A severity of illness, B race/ethnicity, $\mathbf{C}$ language status, and D social vulnerability index are shown. 

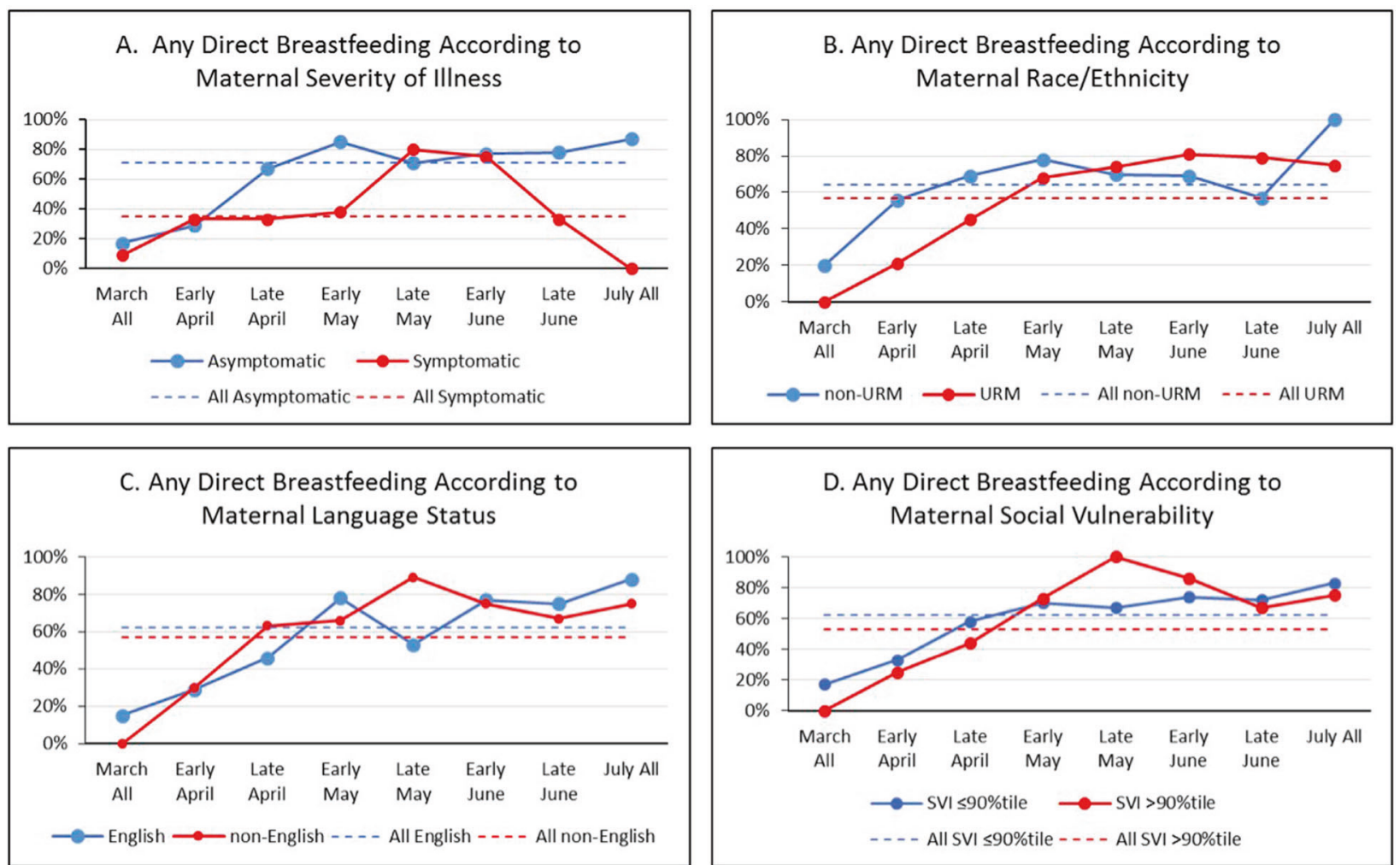

Fig. 5 URM = underrepresented minority (non-Hispanic black or Hispanic of any race); average percent of any direct breastfeeding during the newborn hospitalization over time according to maternal. A severity of illness, B race/ethnicity, C language status, and D social vulnerability index are shown.

toward patients by health care providers occur [38] and that implementing strategies to address social disparities in SARS-CoV2 infection and vaccination are crucial.

Strengths of our quality initiative include use of both practice surveys and tracking of individual-level dyads among centers serving a large proportion of communities with COVID-19 to assess changes in perinatal hospital practice outcomes over time. We also tracked outcomes by several social factors to assess equity in care. Because of the rapid changes and relatively short duration of this initiative, we were unable to track detailed accounts of plan-do-study-act cycles by teams. Further, we tracked outcomes of mothers with positive SARS-CoV-2 testing around the time of birth only, such that we were unable to capture outcomes of mothers with positive SARS-CoV-2 testing earlier in pregnancy. We did not obtain data on patient reported measures, which may have yielded more information about families' experiences during the perinatal hospitalization.

\section{CONCLUSION}

We successfully leveraged our existing PQC structure to disseminate emerging data and build consensus to inform rapid, statewide changes in COVID-19 perinatal practices, suggesting a novel use of PQCs during health crises when best practices are uncertain and quickly evolving. We found that an initial highly protective strategy of cesarean delivery with maternal-infant separation and temporary avoidance of direct breastfeeding gradually transitioned to a decrease in cesarean delivery and increase in rooming-in and direct breastfeeding. Our experience further strengthens the rationale for policy support and funding for PQCs at the state-level. Because perinatal care quality may vary by social factors, which may widen or persist with quality improvement interventions, we proactively examined changes in perinatal care practices according to social factors to monitor for any potential inequities. While tracking of social factors in perinatal quality improvement still remains uncommon, it is crucial to do so in order to address the potential for social inequities in perinatal care delivery.

\section{REFERENCES}

1. Yu N, Li W, Kang Q, Xiong Z, Wang S, Lin X, et al. Clinical features and obstetric and neonatal outcomes of pregnant patients with COVID-19 in Wuhan, China: a retrospective, single-centre, descriptive study. Lancet Infect Dis. 2020;20:559-64.

2. DiGirolamo AM, Grummer-Strawn LM, Fein SB. Effect of maternity-care practices on breastfeeding. Pediatrics. 2008;122:S43-S49.

3. Perinatal Quality Collaborative Guide Working Group. Developing and Sustaining Perinatal Quality Collaboratives. 2016. https://www.cdc.gov/reproductivehealth/ maternalinfanthealth/pdf/Best-Practices-for-Developing-and-SustainingPerinatal-Quality-Collaboratives tagged508.pdf. Accessed 9 Nov 2018.

4. Gupta M, Donovan EF, Henderson Z. State-based perinatal quality collaboratives: pursuing improvements in perinatal health outcomes for all mothers and newborns. Semin Perinatol. 2017;41:195-203.

5. Parker MG. Massachusetts perinatal COVID-19 practice survey results April 2020. https://5d529773-b98a-4c63-93e5-85485fbbebad.filesusr.com/ugd/ 55ea1e 4efcbfdd37b746729da38423d2c2aaef.pdf. Accessed 4 Dec 2020.

6. Parker MG, Burnham LA, Melvin P, Singh R, Lopera AM, Belfort MB, et al. Addressing disparities in mother's milk for VLBW infants through statewide quality improvement. Pediatrics. 2019; 144. https://doi.org/10.1542/peds.2018-3809.

7. Hwang SS, Melvin P, Diop H, Settle M, Mourad J, Gupta M. Implementation of safe sleep practices in Massachusetts NICUs: a state-wide QI collaborative. J Perinatol. 2018;38:593-9.

8. Wachman EM, Houghton M, Melvin P, Isley BC, Murzycki J, Singh R, et al. A quality improvement initiative to implement the eat, sleep, console neonatal opioid withdrawal syndrome care tool in Massachusetts' PNQIN collaborative. J Perinatol. 2020;40:1560-9. 
9. Profit J, Gould JB, Bennett M, Goldstein BA, Draper D, Phibbs CS et al. Racial/ ethnic disparity in NICU quality of care delivery. Pediatrics. 2017; 140. https://doi. org/10.1542/peds.2017-0918.

10. Sigurdson K, Mitchell B, Liu J, Morton C, Gould JB, Lee HC et al. Racial/ethnic disparities in neonatal intensive care: a systematic review. Pediatrics. 2019; 144. https://doi.org/10.1542/peds.2018-3114.

11. Palau MA, Meier MR, Brinton JT, Hwang SS, Roosevelt GE, Parker TA. The impact of parental primary language on communication in the neonatal intensive care unit. J Perinatol. 2019;39:307-13.

12. Lion KC, Raphael JL. Partnering health disparities research with quality improvement science in pediatrics. Pediatrics. 2015;135:354-61.

13. Green AR, Tan-McGrory A, Cervantes MC, Betancourt JR. Leveraging quality improvement to achieve equity in health care. Jt Comm J Qual Patient Saf. 2010;36:435-42.

14. Masschusetts Department of Public Health. Coronavirus Disease 2019 (COVID-19) Cases in MA As of March 15, 2020. 2020. https://www.mass.gov/files/documents/ 2020/03/15/covid-19-case-report-3-15-2020.pdf. Accessed 9 Apr 2021.

15. Betsy Lehman Center for Patient Safety. https://betsylehmancenterma.gov/. Accessed 15 Dec 2020

16. Institute for Health and Recovery. http://www.healthrecovery.org/. Accessed 15 Dec 2020.

17. HUD-USPS ZIP Code Crosswalk Data API Now Available | HUD USER. https://www. huduser.gov/portal/elist/2019-january_29.html. Accessed 6 Apr 2021.

18. CDC Social Vulnerability Index. 2020. https://www.atsdr.cdc.gov/placeandhealth/ svi/index.html. Accessed 4 Dec 2020.

19. Chen $D$, Yang $H$, Cao $Y$, Cheng $W$, Duan $T$, Fan $C$, et al. Expert consensus for managing pregnant women and neonates born to mothers with suspected or confirmed novel coronavirus (COVID-19) infection. Int J Gynaecol Obstet. 2020;149:130-6.

20. NeoQIC COVID-19 Response. 2020. https://www.neoqicma.org/covid-19.

21. PNQIN MPQC and the Betsy Lehman Center for Patietn Safety COVID-19 and Pregnancy in Massachusetts: Virtual Town Hall Series. 2020. https://www. pnqinma.org/pnqin-covid-19-response. Accessed 4 Dec 2020.

22. Provost Lloyd P.; Murray, Sandra K. The healthcare data guide: learning from data for improvement. Jossey-Bass, 2011.

23. State Perinatal Quality Collaboratives | Perinatal | Reproductive Health | CDC. 2020. https://www.cdc.gov/reproductivehealth/maternalinfanthealth/pqc-states. html. Accessed 6 Apr 2021.

24. Lee HC, Bennett MV, Crockett M, Crowe R, Gwiazdowski SG, Keller $H$, et al Comparison of collaborative versus single-site quality improvement to reduce NICU length of stay. Pediatrics. 2018; 142. https://doi.org/10.1542/peds.20171395.

25. Lapcharoensap W, Bennett MV, Powers RJ, Finer NN, Halamek LP, Gould JB, et al. Effects of delivery room quality improvement on premature infant outcomes. J Perinatol. 2017;37:349-54.

26. Zhu H, Wang L, Fang C, Peng S, Zhang L, Chang G, et al. Clinical analysis of 10 neonates born to mothers with 2019-nCoV pneumonia. Transl Pediatr. 2020;9:51-60.

27. Schwartz DA. An analysis of 38 pregnant women with COVID-19, their newborn infants, and maternal-fetal transmission of SARS-CoV-2: maternal coronavirus infections and pregnancy outcomes. Arch Pathol Lab Med. 2020;144:799-805.

28. COVID-19 vaginal delivery - a case report-Lowe - 2020-Australian and New Zealand Journal of Obstetrics and Gynaecology-Wiley Online Library. https:// obgyn.onlinelibrary.wiley.com/doi/10.1111/ajo.13173. Accessed 7 Dec 2020.

29. Ferrazzi E, Frigerio L, Savasi V, Vergani P, Prefumo F, Barresi S, et al. Vaginal delivery in SARS-CoV-2-infected pregnant women in Northern Italy: a retrospective analysis. BJOG: Int J Obstet Gynaecol. 2020;127:1116-21.

30. London V Jr, McLaren R Jr, Atallah F, Cepeda C, Mccalla S, Fisher N, et al. The relationship between status at presentation and outcomes among pregnant women with COVID-19. Am J Perinatol. 2020;37:991-4.

31. Knight M, Bunch K, Vousden N, Morris E, Simpson N, Gale C, et al. Characteristics and outcomes of pregnant women admitted to hospital with confirmed SARS-
CoV-2 infection in UK: national population based cohort study. BMJ. 2020;369: $\mathrm{m} 2107$.

32. FAQs: Management of infants born to mothers with suspected or confirmed COVID-19. 2020. https://services.aap.org/en/pages/2019-novel-coronavirus-covid19-infections/clinical-guidance/faqs-management-of-infants-born-to-covid-19mothers/. Accessed 5 Jun 2020.

33. Puopolo $\mathrm{KMH}$, Hudak ML, Kimberlin DW, Cummings J. Initial guidance: management of infants born to mothers with COVID-19. 2020. https://services.aap. org/en/pages/2019-novel-coronavirus-covid-19-infections/clinical-guidance/faqsmanagement-of-infants-born-to-covid-19-mothers/. Accessed 5 May 2020.

34. Coronavirus Disease 2019: consideration for inpatient obstetric healthcare settings. Centers for Disease Control. 2020. https://www.cdc.gov/coronavirus/2019ncov/hcp/inpatient-obstetric-healthcare-guidance.html. Accessed 4 May 2020.

35. Coronavirus Disease 2019 (COVID-19): Considerations for Inpatient Obstetric Healthcare Settings. 2020. https://www.cdc.gov/coronavirus/2019-ncov/hcp/ inpatient-obstetric-healthcare-guidance.html. Accessed 20 Aug 2020.

36. Halscott $T$, Vaught J. Management considerations for pregnant patients with COVID-19. 2020. https://s3.amazonaws.com/cdn.smfm.org/media/2336/ SMFM_COVID_Management_of_COVID_pos_preg_patients_4-30-20_final. pdf (accessed 11 Jan 2020).

37. Alcendor DJ. Racial disparities-associated COVID-19 mortality among minority populations in the US. J Clin Med 2020; 9. https://doi.org/10.3390/jcm 9082442

38. Hall WJ, Chapman MV, Lee KM, Merino YM, Thomas TW, Payne BK, et al. Implicit racial/ethnic bias among health care professionals and its influence on health care outcomes: a systematic review. Am J Public Health. 2015;105:e60-76.

\section{ACKNOWLEDGEMENTS}

We wish to thank all the participating teams and state stakeholders of the Perinatal Neonatal Quality Improvement Collaborative of Massachusetts. This was supported in part by W.K. Kellogg Foundation (PI Parker; P0131665). The funder/sponsor did not participate in this work.

\section{AUTHOR CONTRIBUTIONS}

Parker and Sullivan drafted the original manuscript, conceived and designed the study, and contributed to data acquisition and analysis. Belfort, Melvin, Angelidou conceived and designed the study, contributed to data acquisition and analysis, preparation of the manuscript. Peaceman and Shui, Vaidya, Singh, Bartolome, Patrizi, Culic, Yanni, Chaudhary, and Goldfarb contributed to data acquisition and analysis; reviewed and revised the manuscript for intellectual content. Gupta and Hudak critically reviewed and revised the manuscript for important intellectual content. All authors approved the final manuscript as submitted and agree to be accountable for all aspects of the work.

\section{COMPETING INTERESTS}

The authors declare no competing financial interests.

\section{ADDITIONAL INFORMATION}

Correspondence and requests for materials should be addressed to K.S.

Reprints and permission information is available at http://www.nature.com/ reprints

Publisher's note Springer Nature remains neutral with regard to jurisdictional claims in published maps and institutional affiliations. 